\title{
Developing Affective Communication Skills in a Clinical Laboratory Sciences Program at a Small Liberal Arts College
}

This study evaluated the ability of simulated laboratory activities to develop affective skills, especially oral communication. Surveys were administered to students at the start and end of a clinical chemistry laboratory course at Nazareth College, a primarily liberal arts college that is not attached to a major medical center. These students included both clinical laboratory science majors and non-majors. Survey questions asked students to use a Likert scale to score their preparedness to properly identify patients, verbally communicate critical results, and discuss pre-analytical challenges that affect specimen integrity. During the course, students evaluated mock laboratory results and were required to orally discuss pre-analytical and postanalytical issues with a volunteer role-playing as an emergency department physician. Data indicates statistically significant $(p<0.5)$ increases in mean scores, comparing surveys before and after the course. The smallest increase in mean scores was seen in preparedness to discuss pre-analytic errors with healthcare team members (2.56 increased to 3.79), while the largest increase was seen in preparedness to orally report critical results (2.81 to 4.57$)$. Based on these findings, it appears that the laboratory activities succeeded in boosting students' confidence and perceptions of preparedness to communicate successfully with healthcare team members. 